\title{
Clinical Evaluation of Creatine Kinase and Aspartate Aminotransferase for Monitoring Muscle Effort in Working Dogs in Different Simulated Fieldworks
}

\author{
Giuseppe Spinella ${ }^{1}$, Simona Valentini ${ }^{1, *}$, Vincenzo Musella ${ }^{2}$, Enrico Bortolotti ${ }^{3}$ and Mirella Lopedote ${ }^{3}$ \\ 1 Department of Veterinary Medical Sciences, University of Bologna, Ozzano dell'Emilia, 40064 Bologna, Italy; \\ giuseppe.spinella@unibo.it \\ 2 Department of Health Sciences, University of Catanzaro, 88100 Catanzaro, Italy; musella@unicz.it \\ 3 Clinica Veterinaria San Michele, 38010 Grumo di San Michele all'Adige, Italy; enricobor@me.com (E.B.); \\ mirella.lopedote@yahoo.com (M.L.) \\ * Correspondence: simona.valentini@unibo.it; Tel.: +39-051-2097530
}

check for updates

Citation: Spinella, G.; Valentini, S.; Musella, V.; Bortolotti, E.; Lopedote, M. Clinical Evaluation of Creatine Kinase and Aspartate

Aminotransferase for Monitoring Muscle Effort in Working Dogs in Different Simulated Fieldworks. Animals 2021, 11, 1879. https:// doi.org/10.3390/ani11071879

Academic Editor: Yves Samoy

Received: 20 May 2021

Accepted: 21 June 2021

Published: 24 June 2021

Publisher's Note: MDPI stays neutral with regard to jurisdictional claims in published maps and institutional affiliations.

Copyright: (c) 2021 by the authors. Licensee MDPI, Basel, Switzerland. This article is an open access article distributed under the terms and conditions of the Creative Commons Attribution (CC BY) license (https:// creativecommons.org/licenses/by/ $4.0 /)$.
Simple Summary: Search and rescue dogs are increasingly involved in finding survivors during catastrophic events. The need to investigate the possible physical conditions that can lead to disabling pathologies is urgent. In this clinical study, muscular effort was investigated through the evaluation of two muscle enzymes: creatine kinase and aspartate aminotransferase. The results show that effective conditioning supports submaximal activity (about $20 \mathrm{~min}$ ), without any specific muscular enzymatic alteration.

Abstract: The clinical profiles of muscle biomarkers (Creatine Kinase-CK-and Aspartate Aminotransferase-AST) performed during training may help in determining the fitness level of dogs and their potentiality to perform specific activities. This study investigated the potential variations of physiological parameters and muscular biomarkers in trained search and rescue dogs during search activity in two different areas. The aim was to verify the absence of any muscular enzymes after $20 \mathrm{~min}$ of search activity. The variations of physiological parameters (pulse rate; respiratory rate; rectal body temperature) and skeletal muscular biomarkers (CK and AST) were evaluated before and after search activity. Twenty-three trained dogs met the inclusion criteria and were divided into two groups. One group experienced search activity in a well-known area, while the second one in a similar, but unknown, area. The results for physiological parameters and skeletal muscular biomarkers values showed no significant differences between the two groups $(p>0.05)$, confirming that an effective conditioning protects against enzymatic alteration during a 20 min duration of submaximal activity.

Keywords: search and rescue activity; creatine kinase; aspartate aminotransferase; pulse rate; respiratory rate; rectal temperature; dog

\section{Introduction}

Search and rescue dogs (SRDs) have a great social impact due to their invaluable help in finding survivors during catastrophic events, such as earthquakes. Specific skills, such as obedience, handler bonding and human airscent detection are fundamental peculiarities of SRDs, but these athlete dogs also require adequate physical fitness to cover large areas of land when searching, often in adverse conditions [1,2]. In veterinary sports medicine, it is of great relevance to monitor canine training and conditioning to avoid anomalous skeletal muscular response, likely a prodromal state for the onset of muscle fatigue. Muscle fatigue is defined as a decrease in maximal force or power production in response to contractile activity [3]. Fatigue can originate at different levels of the motor pathway and is usually divided into central and peripheral components. Muscle fatigue is 
a commonly experienced phenomenon that limits athletic performance and other strenuous or prolonged activity [4]. However, the level of change in physiological, haematological and metabolic parameters occurring with exercise are dependent on several factors that include the typology of work and the grade of canine training as well as environmental factors [5]. The clinical profiles of muscle biomarkers performed during training may help in determining the fitness level of dogs and their potentiality to perform specific activities [5]. The most investigated biomarkers have been creatine kinase (CK), aspartate aminotransferase (AST), myoglobin and serum lactate concentrations. In veterinary sports dogs and SDRs, physiological parameters have been widely investigated, but scientific information on skeletal muscle biomarkers has been reported less frequently, generally in racing greyhounds or sled dogs, and less frequently, in SRDs [1,2,5-7]. Generally, a mild increase of specific muscle biomarkers was observed in dogs after prolonged endurance exercise and high-intensity racing. Longer and/or more intense exercise usually leads to an increase in significant changes, while less intense sessions may not have a significant impact. However, in endurance dogs, a targeted training programme may correctly prepare the dog for competition without any skeletal muscle injury [5]. To the authors' knowledge, no publication has investigated the skeletal muscular response during submaximal exercise (20 min of duration) in SRDs, comparing the search activity in known or unknown working areas.

The aim of this study was to verify any potential variations of physiological parameters (pulse rate-PR; respiratory rate-RR; body temperature-BT) and skeletal muscular biomarkers (CK and AST) in trained SRDs during search activity in two different areas. One group experienced a known space of search (previously used for dog training), while the other group searched in an unknown area approached for the first time by the dogs (unknown field).

Our hypothesis was that well-trained dogs do not experience any permanent muscular damage during a submaximal search exercise (about $20 \mathrm{~min}$ ), regardless of whether approaching a known or unknown search area.

\section{Materials and Methods}

Ethical approval for this study was obtained from the Animal Welfare Committee of the University of Bologna, in accordance with Italian DL 26/2014 (Project ID 914).

A team of 24 SRDs was involved in this observational study. All animals were skilled in search and rescue activity and fed with a commercial food for adult dogs. All dogs were conditioned under a similar training approach: conditioning included one day per week of obedience activity and two days per week of search activity in a training field. Moreover, each day, the dogs went freely with their owners along a $6 \mathrm{~km}$ route. The inclusion criteria were the completion of the search activity and absence of clinical and blood biochemical alterations before the search activity.

\subsection{Clinical and Laboratory Monitoring}

All dogs were clinically monitored by two licensed doctors in veterinary medicine before the trial to ensure a good state of health and suitability to perform the scheduled search activity. No dogs received any medication or drugs within 15 days before the search activity. Dog signalments and physiological parameters (pulse rate, respiratory rate and rectal body temperature) were recorded for all dogs at rest, in order to exclude any physiological variation related to environmental factors and the potential excitement state. The pulse rate was detected with palpation of femoral artery and respiratory rate was measured by thoracic visual observation. A first blood sample for CK and AST evaluation was collected $15 \mathrm{~min}$ before search activity by saphenous vein. Immediately after activity, PR and RR were measured; a second blood sample and BT measurement were collected and recorded two hours after the activity. The timetable for the evaluation of muscular enzymes was scheduled two hours after activity in order to ensure that an increase of enzyme 
concentration could be reasonably attributed to prolonged muscle damage secondary to an excessive muscular effort [8].

\subsection{Search Activity and Features of the Search Areas}

The study was created following the regulations of the Italian National Canine Association (Ente Nazionale Cinofilia Italiano-ENCI). The test involved a search by the dog for two missing persons ('victims') in an area from 30,000 to 50,000 square m, depending on the morphology of the land and vegetation. The evaluation parameters for this test were: (a) docility, cooperation of the dog with the handler, prompt and targeted execution of orders, maintaining motivation in the search; (b) intensity in searching, behaviour during the search, temperament, motivation, enthusiasm, physical condition; (c) agility, movement in the search area, facing difficulties; (d) independence in searching; (e) the quality of the tactical choice and its implementation, management of the entire search operation; (f) behaviour of the dog with disruptive walker-ons; (g) finding the 'victim'.

On the day of search activity, the team was randomly divided into two groups. Group 1 searched the well-known area, while Group 2 searched an unknown area. The start of the activity occurred approximately every $30 \mathrm{~min}$ for each dog. A maximum of $20 \mathrm{~min}$ of search time was allowed for each dog to locate the two 'victims' (as required by time imposed by ENCI regulation).

Area n.1 (area already known by dogs of Group 1)-wooded area in Clusone (North of Italy), east side, rich in larch and fir trees with a dense undergrowth with many brambles. Within the search area, there were some clearly visible paths, with the main part of the area located on a natural terrace at an altitude of $549 \mathrm{~m}$ above sea level, which then sloped down on the north side and on the west side to a flat area below. The total height difference of the area was about $50 \mathrm{~m}$.

Area n.2 (area unknown by dogs of Group 2)-located within the pine forest of Clusone, but placed towards Mount Senda, on the west side. A steep, but not impervious, slope rose from 530 to $650 \mathrm{~m}$ altitude and the vegetation was composed of larches and firs with dense undergrowth, also characterised by some patches of brambles. The total difference in height of the area was about $120 \mathrm{~m}$.

During the clinical study, the environmental air temperatures ranged from 18 to $26^{\circ} \mathrm{C}$ with $70 \%$ humidity and a relative wind speed of $7-8 \mathrm{~m} / \mathrm{h}$.

\subsection{Statistical Analysis}

All data were submitted to descriptive (mean \pm standard deviation and median) and analytic statistical analysis for the entire team and specifically, within Groups 1 and 2. All data were previously submitted to the Shapiro-Wilk test for an evaluation of normality distribution. If normal distribution was not reported, physiological and serum parameters were evaluated with the Wilcoxon-Mann-Whitney $t$ test, in order to investigate any significant variation between rest and after the search activity. Significance for all tests was set at $p<0.05$. Statistical analyses were performed using Stata software, version 15 (StataCorp, College Station, TX, USA, 2017).

\section{Results}

Twenty-four dogs were enrolled in this study and all dogs completed the search activity. However, one dog was excluded because of high pre-competition values of AST and CK. The 23 dogs were: five German Shepherds, three Malinois, three Golden Retrievers, three Labradors, three mixed breed dogs, two Border Collies, two Australian Shepherds, one Jack Russel terrier and one Weimaraner. Ten females and two males were included in Group 1, and nine females and two males in Group 2. The mean age of the entire team was $4.6 \pm 2.5$ years (median 5): $5.3 \pm 2.4$ and $3.9 \pm 2.4$ years, respectively in Group 1 and Group 2. 
No significant difference was observed between the two groups for age distribution $(p=0.191)$. Mean PRs (beats per minute), respectively for Groups 1 and 2, were $61.6 \pm 17.5$ and $64.5 \pm 13.8$ at rest, and $114.6 \pm 38.7$ and $99.82 \pm 14.8$ after activity. Mean RRs (breaths per minutes) were $29.7 \pm 14.3$ and $33.6 \pm 19.8$ at rest, and $197.3 \pm 44.3$ and $194.1 \pm 37.1$ after activity, respectively for Groups 1 and 2. Mean BTs were $38 \pm 0.4{ }^{\circ} \mathrm{C}$ and $37.9 \pm 0.4{ }^{\circ} \mathrm{C}$ at rest, and $38.6 \pm 0.6{ }^{\circ} \mathrm{C}$ and $38.8 \pm 0.5^{\circ} \mathrm{C}$ two hours after activity, respectively for Groups 1 and 2. For physiological parameters (PR, RR, BT), no significant differences were observed for variations between the two groups in relation to physical activity; while significant differences were shown for PR, RR and BT before and after activity $(p<0.01)$ for both groups (Figure 1).
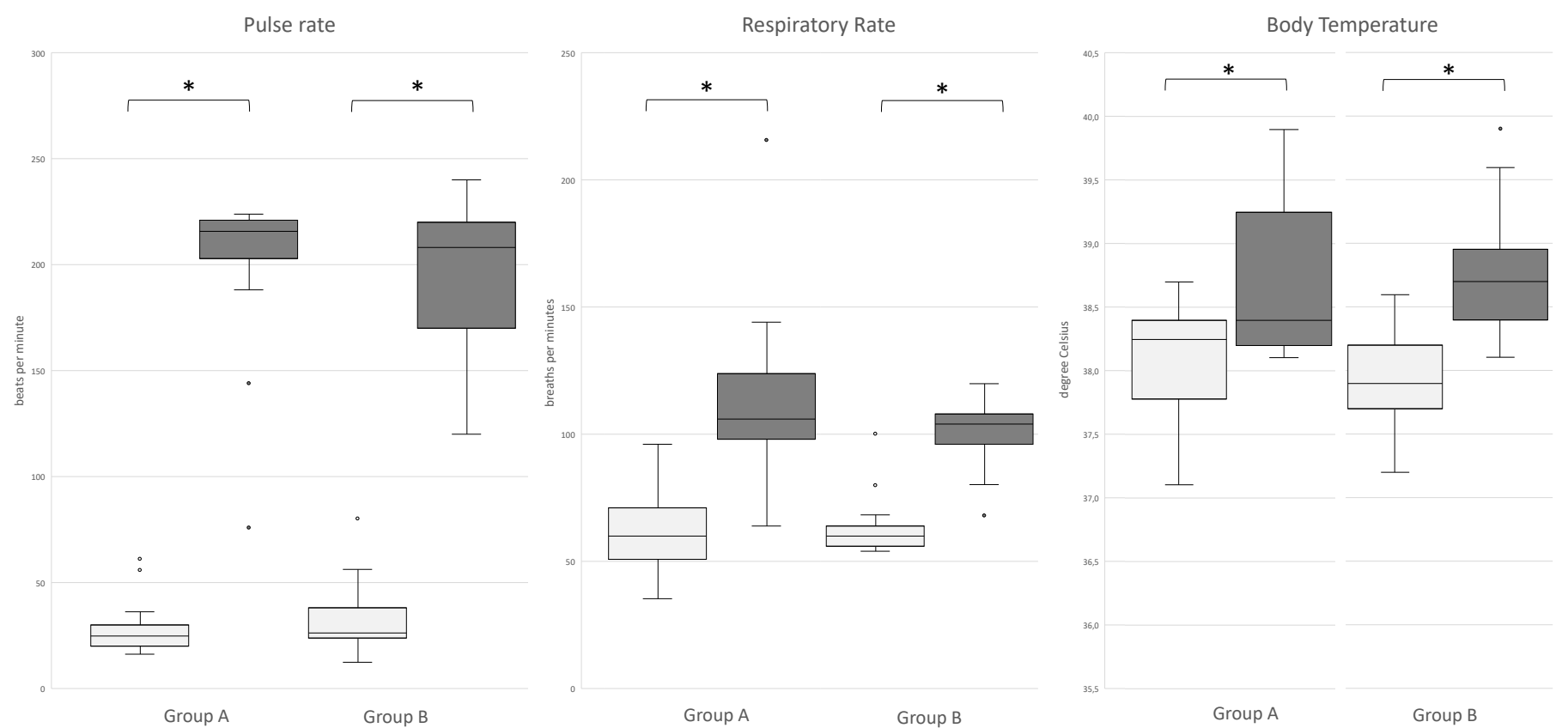

Figure 1. Boxplots of pulse rate, respiratory rate and body temperature $\left({ }^{\circ} \mathrm{C}\right)$ monitored before and after activity, in Group 1 (A) and Group 2 (B). Statistical differences were observed in all three physiological parameters $\left({ }^{*} p<0.05\right)$.

The mean AST values of $34.75 \pm 9.2 \mathrm{U} / \mathrm{L}$ and $31.18 \pm 8.5 \mathrm{U} / \mathrm{L}$ were reported, respectively for Groups 1 and 2 before activity, and $39.42 \pm 8.5 \mathrm{U} / \mathrm{L}$ and $37.9 \pm 11.5 \mathrm{U} / \mathrm{L}$ two hours after activity. Mean CK values of $104.33 \pm 86.1 \mathrm{U} / \mathrm{L}$ and $66 \pm 38.8 \mathrm{U} / \mathrm{L}$ were reported, respectively for Groups 1 and 2 before activity, and $107.42 \pm 65.6$ and $99.82 \pm 65.3$ two hours after activity. For AST and CK values, no significant differences were reported between the groups $(p>0.05)$ (Figure 2). 

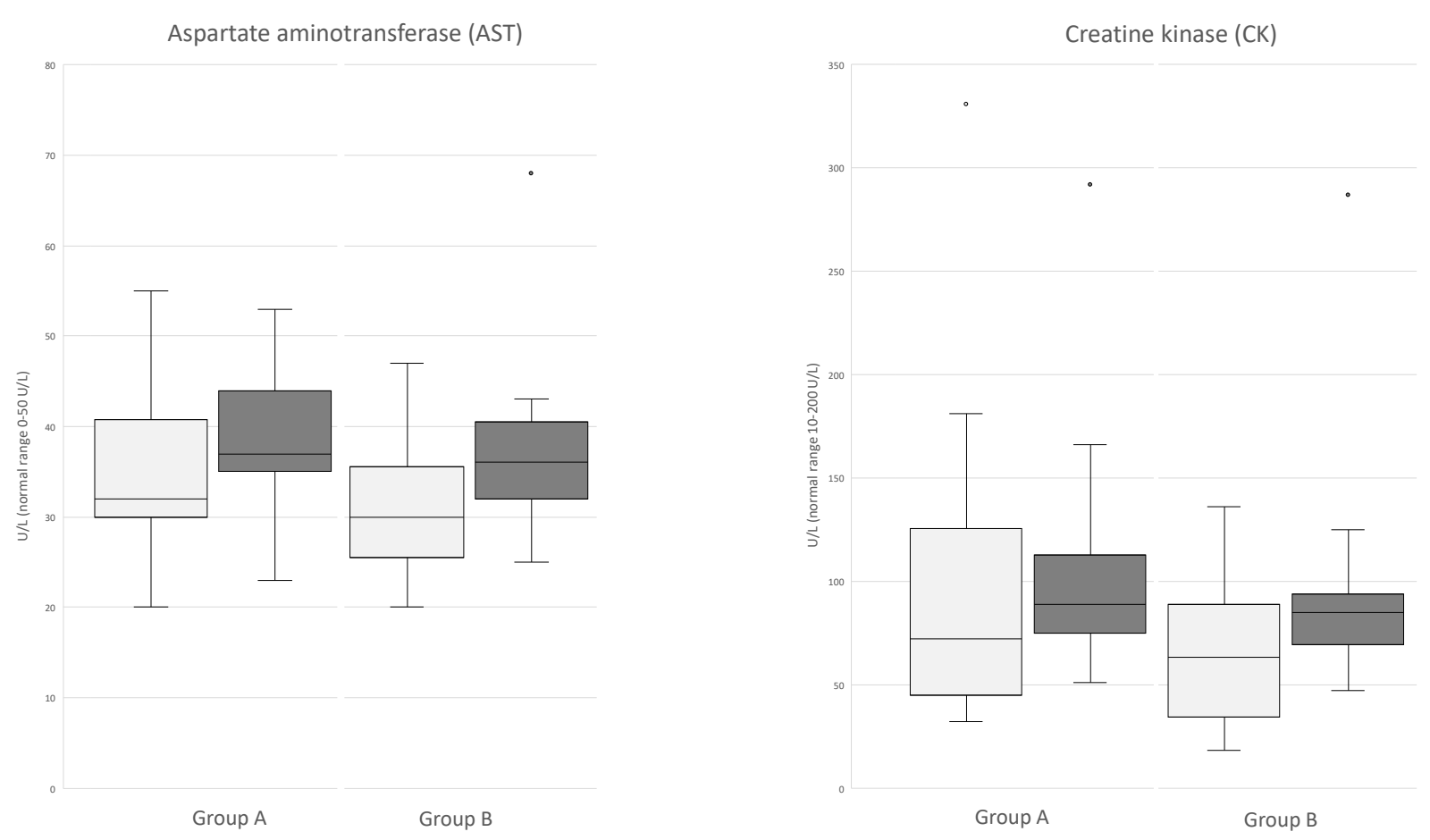

Figure 2. Boxplots of aspartate aminotransferase (AST) and creatine kinase (CK) monitored before and $2 \mathrm{~h}$ after activity in Group 1 (A) and Group 2 (B). No statistical differences were observed.

\section{Discussion}

This study aimed to investigate the absence of muscle damage in well-trained dogs during a submaximal search exercise (about $20 \mathrm{~min}$ ), in two different working areas (known and unknown). The results show that no statistical differences were observed between the two groups and the hypothesis was therefore confirmed.

Both groups reported an increase of PR and RR immediately after activity as well as an increase in BT.

Variations in physiological parameters for working and sports dogs have been investigated during competitive and conditioning activity [1,2,5-8]. Pulse and respiratory rates generally increased after activity in response to increased energy production during exercise and to eliminate the produced heat as a by-product. However, if heart and pulse rates are measured immediately before a competition, a significant increase could be observed compared to rest, as a response to exercise anticipation, also reported as the 'eureka effect' $[2,6,8,9]$. Systematic training is a continuous process that aims to maximise aerobic capacity through weekly exercise sessions, individually prescribed to decrease the risk of musculoskeletal injury [10]. Our investigation showed a BT increase after activity, but this variation was within the physiological range two hours after the activity. Indeed, as previously reported by Matwichuk et al. (1999) in healthy Labrador retrievers, no significant rectal temperature variations were observed in exercising dogs when the environmental temperature ranged between $11-28{ }^{\circ} \mathrm{C}$ [11].

In our study, muscular enzymes results demonstrated a mild increase, but this variation was not statistically different and within the normal range.

Creatine kinase $(\mathrm{CK})$ is the best indicator of striated muscle damage as most of its activity is in skeletal muscle. The increase in plasma CK activity in dogs is due to its leakage through the cell membrane and therefore, is evident in all conditions associated with muscle inflammation, necrosis or degeneration [12]. CK has a half-life of 2-3 h, so high serum activity after two hours could be indicative of recent or active muscle damage.

Aspartate aminotransferase (AST) is also indicative of muscle damage, although it is less organ-specific than CK. AST is also found in the liver. The AST assessment is 
useful, together with the CK assessment, to understand if muscle damage is evolving or resolving [12].

Previous studies on sports dogs revealed that prolonged endurance exercise and highintensity racing induced a mild, but significant, elevation in the skeletal muscle biomarkers, CK and AST $[13,14]$. However, Cerqueira et al. observed that an endurance-training programme (approximately $42-56 \mathrm{~min}$ ) improved the aerobic capacity of dogs without any skeletal or cardiac muscle injury [5]. Working dogs that experienced a maximum of 10 min of search activity for victims, after helicopter transport, reported an increase of CK and AST blood concentrations after search activity, but normal concentrations were detected after two hours from the end of the activity [8]. For this specific reason, our second blood collection was scheduled two hours after activity, to minimise the exercise effect on muscular metabolism and to detect potential muscular damage.

A study conducted by Hinchcliff reported that differences in serum biochemical variables between dogs that completed a race and those that were retired, were not clinically significant. It was therefore not possible to identify any consistent factors that were associated with a failure to complete a race (retired dogs) [15].

\section{Conclusions}

In conclusion, our study aimed to investigate whether prolonged muscle injury occurred following a submaximal search exercise (about $20 \mathrm{~min}$ ) in well-trained SDRs that experienced a search activity in an unknown area, as normally happens in real conditions. All dogs correctly concluded their scheduled activity: potential variations of skeletal muscular biomarkers (CK and AST) were not observed two hours after the activity. Moreover, it could be assumed that the pause of two hours, after the end of the research activity, may be enough for observing the values of CK and AST in physiological ranges and consequently, the dog could be ready for a new work session. However, this latter hypothesis should be investigated with further prospective studies.

Author Contributions: Conceptualization, G.S. and M.L.; methodology, M.L. and E.B.; validation, G.S., S.V. and V.M.; formal analysis, V.M.; writing — original draft preparation, G.S. and S.V. writingreview and editing, G.S. and M.L. All authors have read and agreed to the published version of the manuscript.

Funding: This research received no external funding.

Institutional Review Board Statement: The study was conducted according to the guidelines of the Declaration of Helsinki and approved by Animal Welfare Committee of the University of Bologna, in accordance with Italian DL 26/2014 (Project ID 914). Oral consent was also obtained by each dog owner the day of the activity; a previous email had explained the aim and the methodology of the study.

Data Availability Statement: All data is contained within this article. Interested qualified researchers may request further information by contacting the corresponding author.

Acknowledgments: Authors particularly thanks ARGO dog nucleus of National Alpine Association of Bergamo (Italy).

Conflicts of Interest: The authors declare no conflict of interest.

\section{References}

1. Rovira, S.; Munoz, A.; Benito, M. Effect of exercise on physiological, blood and endocrine parameters in search and res-cue-trained dogs. Vet. Med. 2008, 53, 333-346. [CrossRef]

2. Lopedote, M.; Valentini, S.; Musella, V.; Vilar, J.M.; Spinella, G. Changes in pulse rate, respiratory rate and rectal tempera-ture in working dogs before and after three different field trials. Animals 2020, 10, 733. [CrossRef] [PubMed]

3. Gandevia, S.C. Spinal and Supraspinal Factors in Human Muscle Fatigue. Physiol. Rev. 2001, 81, 1725-1789. [CrossRef] [PubMed]

4. Wan, J.-J.; Qin, Z.; Wang, P.-Y.; Sun, Y.; Liu, X. Muscle fatigue: General understanding and treatment. Exp. Mol. Med. 2017, 49, e384. [CrossRef] [PubMed] 
5. Cerqueira, J.A.; Restan, W.A.Z.; Fonseca, M.G.; Catananti, L.A.; de Almeida, M.L.M.; Junior, W.H.F.; Pereira, G.T.; Carciofi, A.C.; de Camargo Ferraz, G. Intense exercise and endurance-training program influence serum kinetics of muscle and cardi-ac biomarkers in dogs. Res. Vet. Sci. 2018, 121, 31-39. [CrossRef] [PubMed]

6. Alves, J.C.; Santos, A. Physiological, haematological and biochemical shifts in police working dogs during a riot control exer-cise. Comp. Exerc. Physiol. 2016, 12, 193-198. [CrossRef]

7. Spoo, J.W.; Zoran, D.L.; Downey, R.L.; Bischoff, K.; Wakshlag, J.J. Serum biochemical, blood gas and antioxidant status in search and rescue dogs before and after simulated fieldwork. Vet. J. 2015, 206, 47-53. [CrossRef] [PubMed]

8. Diverio, S.; Barbato, O.; Cavallina, R.; Guelfi, G.; Iaboni, M.; Zasso, R.; Di Mari, W.; Santoro, M.M.; Knowles, T.G. A simulated avalanche search and rescue mission induces temporary physiological and behavioural changes in military dogs. Physiol. Behav. 2016, 163, 193-202. [CrossRef] [PubMed]

9. McGowan, R.T.S.; Rehn, T.; Norling, Y.; Keeling, L.J. Positive affect and learning: Exploring the "Eureka Effect" in dogs. Anim. Cogn. 2014, 17, 577-587. [CrossRef] [PubMed]

10. Pellegrino, F.J.; Risso, A.; Vaquero, P.G.; Corrada, Y.A. Physiological parameter values in greyhounds before and after highintensity exercise. Open Vet. J. 2018, 8, 64-67. [CrossRef] [PubMed]

11. Matwichuk, C.L.; Taylor, S.; Shmon, C.L.; Kass, P.H.; Shelton, G.D. Changes in rectal temperature and hematologic, biochemical, blood gas, and acid-base values in healthy Labrador Retrievers before and after strenuous exercise. Am. J. Vet. Res. 1999, 60, 88-92.

12. Lucas, V.; Barrera, R.; Duque, F.J.; Ruiz, P.; Zaragoza, C. Effect of exercise on serum markers of muscle inflammation in Spanish Greyhounds. Am. J. Vet. Res. 2015, 76, 637-643. [CrossRef] [PubMed]

13. Tharwat, M.; Al-Sobayil, F.; Buczinski, S. Influence of racing on the serum concentrations of acute-phase proteins and bone metabolism biomarkers in racing greyhounds. Vet. J. 2014, 202, 372-377. [CrossRef] [PubMed]

14. Vlasakova, K.; Lane, P.; Michna, L.; Muniappa, N.; Sistare, F.D.; Glaab, W.E. Response of Novel Skeletal Muscle Biomarkers in Dogs to Drug-Induced Skeletal Muscle Injury or Sustained Endurance Exercise. Toxicol. Sci. 2017, 156, 422-427. [CrossRef] [PubMed]

15. Hinchcliff, K. Performance failure in Alaskan sled dogs: Biochemical correlates. Res. Vet. Sci. 1996, 61, 271-272. [CrossRef] 